\title{
ON MAPPINGS FROM COMPLEXES INTO THE COMPLEX PROJECTIVE SPACE
}

\author{
HideKaZU WADA \\ (Received October 20, 1951)
}

1. Introduction. L. Pontrjagin [2] classified the mappings from any three-dimensional complex into the 2 -sphere, and also classified the algebraically inessential mappings from any arbitrary dimensional complex into the 2 -sphere. In this paper, we shall treat a generalization of his results. Namely, for two mappings $f, g$ from the $(2 n+1)$-dimensional complex in the complex $n$-dimensional complex projective space, whose partial mappings are the same in the 2 -skeleton, an integral $(2 n+1)$-cocycle, which we shall call the separation obstruction $d^{2 n+1}(f, g)$, can be defined; and the necessary and sufficient condition that they are homotopic is that there exists an integral 1-cocycle $w^{1}$ such that $d^{2 n+1}(f, g) \sim 2^{n} w^{1} \cdot\left(f^{*} s^{2}\right)^{n}$, where the product of the right-hand side of this cohomology means the cup product of cocycles based on the usual products of integers of coefficients. When $n=1$, this result is nothing but the theorem of L. Pontrjagin [2] and proved by N. E. Steenrod [3] by another method. Here we shall prove the result by the method of N. E. Steenrod.

Secondly, we shall show, that the classes of algebraically inessential mappings from the arbitrary dimensional complex. whose 1-cohomology group with the integral coefficient vanishes, into the complex projective space, are in 1-1 correspondence to the classes of mappings from that complex into the $(2 n+1)$-dimensional sphere. But the author can not avoid here the cohomological assumption in the case $n>1$. We shall prove this last half by the theory of fibre bundles.

2. Complex projective space. Let an oriented $(2 n+1)$-sphere $S^{2 n+1}$ be represented by the coordinates of the complex numbers $\left(z_{0}, z_{1}, \ldots, z_{n}\right)$ as follows :

$$
\sum_{k=0}^{n} z_{k} z_{i b}=1 .
$$

Now, let us call two points $\left(z_{1}, \ldots, z_{n}\right)$ and $\left(z_{0}^{\prime}, \ldots, z_{n}^{\prime}\right)$ on $S^{2 n+1}$ to be equivalent provided that there exists a real number $\theta$ such that :

$$
z_{k}=\exp (\sqrt{-1} \theta) z_{k}^{\prime} \quad(k=0,1, \ldots, n) .
$$

Then the factor space defined by this equivalence relation, is the complex projective space $P(n)$; and it is well known [4; p. 108] that $S^{2 n+1}$ is an orientable circle bundle ${ }^{1)}$ over $P(n)$, and that the canonical mapping $p_{n}: S^{z n+1} \rightarrow P(n)$ is the

1) As for fiber bundles, we shall mainely use the definitions and the notations of [4]. 
projection of the bundle. In the sequel, we shall usually represent the euclidean coordinates by $\left(z_{0}, \ldots, z_{n}\right)$, and the homogeneous coordinates of $P(n)$ by $\left[z_{0}, \ldots, z_{n}\right]$.

$$
\begin{array}{ll}
\text { Lemma } 1 . & \pi_{k}(P(n)) \approx \pi_{k}\left(S^{2 n+1}\right) \\
& \pi_{2}(P(n)) \approx J: \text { infinite cyclic group. }
\end{array} \quad(k=0,1,3, \ldots),
$$

In the first isomorphism we have denoted by $\pi_{0}(P(n)) \approx 0$ the arcwise connectedness of $P(n)$.

Proof. $\pi_{0}(P(n)) \approx \pi_{1}(P(n)) \approx 0$ follows from the covering homotopy theorem [4; p. 54], the arcwise connectedness of $S^{2 n+1}$ and $S^{1}$, and the simply connectedness of $S^{2 n+1}$.

Now, let the fibre over $e^{0}=[1,0, \ldots, 0] \in P(n)$ be $S_{0}^{1}$, then we obtain the following exact homotopy sequence of the fibre bundle $[4 ;$ p. 91$]$ :

$$
\cdots \rightarrow \pi_{k}\left(S_{0}^{1}\right) \rightarrow \pi_{k}\left(S^{2 n+1}\right) \rightarrow \pi_{k}(P(n)) \rightarrow \pi_{k-1}\left(S_{0}^{1}\right) \rightarrow \ldots \rightarrow \pi_{1}\left(S^{2 n+1}\right) .
$$

Then the exactness of (2.1) asserts the conclusions of the rest of the proof. Q. E. D.

Next, let $S_{*}^{3}$ be a great 3 -sphere in $S^{2 n+1}$ represented by the coordinates $\left(\tilde{z}_{1}, z_{1}, 0, \ldots 0\right)$; then $p_{n}\left(S_{*}^{3}\right)=S_{*}^{2}$ represents a complex projective line $P(1)$ in $P(n)$, and the mapping $p_{n} \mid S_{*}^{3}$, namely the partial mapping on $S_{*}^{3}$, is well known to have unity as its Hopf's invariant $[4 ;$ p. 113]. Also, it is easily verified that the generator of $\pi_{2}(P(n)) \approx J$ is respresented by the identical mapping of $S_{*}^{2}$, and that the generator of $\pi_{2^{n+1}}(P(n)) \approx J$ is $p_{n}$ itself.

Now, let $E^{2 n}$ be a $2 n$-cell and $\dot{E}^{2 n}$ be its boundary; and let the continuous mapping $q:\left(E^{2 n}, \dot{E}^{2 n}\right) \rightarrow(P(n), P(n-1))$ be defined by the formula :

$$
q\left(z_{0}, \ldots, z_{n-1}\right)=\left[z_{0}, z_{1}, \ldots, z_{n-1},\left(1-\sum_{k=0}^{n-1} z_{k} z_{k}\right)^{1 / 2}\right],\left(\sum_{k=0}^{n-1} z_{k} z_{k} \leqq 1\right)
$$

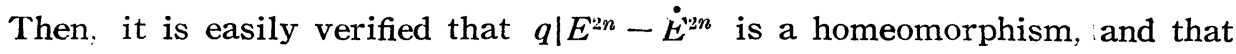
$q \mid \dot{E}^{2 n}=p_{n-1}$. Therefore, we can regard $P(n)-P(n-1)$ as an open $2 n$-cell $\boldsymbol{e}^{2 ! n}$, and consequently, we obtain the following cellular decomposition of $P(n)$ :

$$
P(n)=\bigcup_{k=0}^{n} e^{2 i c} .
$$

Hence the cohomology groups of $P(n)$ are verified to be:

$$
\begin{array}{ll}
H^{2 \prime}(P(n), J) \approx J & (k=0,1, \ldots, n), \\
H^{*}(P(n), J) \approx 0 & \text { otherwise. }
\end{array}
$$

Obviously $e^{0} \in S_{*}^{2}, \quad e^{2}=S_{*}^{2}-e^{0}$.

3. Mappings from the complex into $P(n)$. Now, let $K$ be a finite, geometrical cellular complex, $K^{k}$ be its $k$-skeleton, and $L$ be the subcomplex of $K$; and, we shall denote $K^{s}+L$ by $K^{k}$.

Next, the continuous mapping $f: K \rightarrow P(n)$ is said to be standard, provided 


$$
f\left(K^{1}\right)=e^{0}, \quad f\left(K^{2}\right) \subset S_{*}^{2} .
$$

Then, Lemma 1 and the homotopy extension theorem assert the existence of a standard mapping $f: K \rightarrow P(n)$ homotopic to the given continuous mapping. Let now $s^{2}$ be a generating cocycle of $H^{2}(P(n), J)$, namely such cocycle that $s^{2}\left(e^{2}\right)=1$. Then, for a standard mapping $f: K \rightarrow P(n)$, the cocycle $f^{*} s^{2}$, which takes an integer of $\pi_{2}(P(n)) \approx J$ represented by the mapping $f:\left(\sigma^{2}\right.$, $\left.\dot{\sigma}^{2}\right) \rightarrow\left(S_{*}^{2}, e^{0}\right)$ for an arbitrary cell $\sigma^{2} \subset K$, is obtained; in this case, when $f$ is homotopic to the given continuous mapping, the cohomology class containing $f^{*} s^{2}$ is independent of the choice of the standard mapping $f$.

Next, for a continuous mapping $f: \overline{K^{2} n+1} \rightarrow P(n)$, an integral $(2 n+2)$ cocycle $c^{2 n+2}(f)$ in $K-L$, the obstruction can be obtained ; namely, such cocycle that takes an integer of the element of $\pi_{2 n+1}(P(n)) \approx J$ represented by $f \mid \dot{\sigma}^{2 n+2}$ for any $(2 n+2)$-cell $\sigma^{2 n+2}$ in $K$. define

Again, let $f, g: \bar{K}^{2 n+1} \rightarrow P(n), f|L=g| L$ be standard mappings. If we

$$
(f-g)^{*} z^{*}=f^{*} z^{k}-g^{*} z^{k}
$$

for any $k$-cocycle $z^{3}$ in $P(n)$, with the coefficient $J$, then, it is easily seen that $(f-g)^{*} z^{k}$ is a cocycle in $K-L$, and that the operation $(f-g)^{*}$ induces the homomorphism for the cohomology groups $[3, \S 22]$.

$$
(f-g)^{*}: H^{*}(P(n), J) \rightarrow H^{k}(K-L, J),
$$

denoting by the same symbol $(f-g)^{*}$ the correspondence between the cohomology classes. Then, the main purpose in this section is to prove the following theorem :

Theorem 1. Let $f, g: \bar{K}^{2 n+1} \rightarrow P(n), f|L=g| L$ be standard mappings. Then we get

$$
\begin{gathered}
\left\{c^{2 n+2}(f)\right\}-\left\{c^{2 n+2}(g)\right\}=\sum_{k=0}^{n} 2^{k}\left(\begin{array}{l}
n \\
k
\end{array}\right)\left\{\lambda^{2}\right\}^{n+1-} .\left\{g^{*} s^{2}\right\}^{k}{ }^{2)}, \\
\left(\lambda^{2}=(f-g)^{*} s^{2}\right)
\end{gathered}
$$

where the powers and the products in the right-hand side show the cup products, hased on the usual products of integers of coefficients.

Proof. From Lemma $1, f, g$ can be extended uniquely to $f^{\prime}, g^{\prime}: \overline{K^{2 n+2}} \rightarrow P$ $(n+1)$ within the homotopies relative to $\bar{K}^{2 n+1}$, and for an arbitrary $(2 n+2)$-cell $\sigma^{2 n+2} \subset K, \quad c^{2 n+2}(f) \sigma^{2 n+2}$ and $c^{2 n+2}(g) \sigma^{2 n+2}$ take the integers of $\pi_{2 n+2}(P(n+1), P(n)) \approx J$ represented by $f^{\prime} \mid \sigma^{2 n+2}$ and $g^{\prime} \mid \sigma^{2 n+2}$ respectively. They are obviously same as $f^{\prime *} t^{2 n+2}\left(\sigma^{2 n+2}\right)$ and as $g^{\prime \prime} t^{2 n+2}\left(\sigma^{2 n+2}\right)$ respectively, by denoting by $t^{2 n+2}$ the generating cocycle of $P(n+1)$ such that $t^{2 n+2}\left(e^{2 n+2}\right)=1$. (Cf, §1). Therefore we obtain analogously to [3]

2) $\{u\}$ means the relative and absolute cohomology class containing the corresponding cocycle $u$. In this case, $g^{*} s^{2}$ is an absolute one, and the others are relative ones. 


$$
\begin{aligned}
\left\{c^{2 n+2}(f)\right\}-\left\{c^{2 n+2}(g)\right\}= & \left\{c^{2 n+2}(f)-c^{2 n+2}(g)\right\}=\left\{\left(f^{\prime}-g^{\prime}\right)^{*} t^{2 n+2}\right\} \\
& =\left(f^{\prime}-g^{\prime}\right)^{*}\left\{t^{3 n+2}\right\}=\left(f^{\prime}-g^{\prime}\right)^{*}\left\{s^{2}\right\}^{n+1} .
\end{aligned}
$$

On the other hand, N.E.Steenrod $[3,(22.8)]$ showed that $\left(f^{\prime}-g^{\prime}\right)^{*}$ operates on the cup product as follows

$$
\begin{aligned}
& \left(f^{\prime}-g^{\prime}\right)^{*}(\{u\} \cdot\{v\})=\left(\left(f^{\prime}-g^{\prime}\right)^{*}\{u\}\right) \cdot\left(\left(f^{\prime}-g^{\prime}\right)^{*}\{v\}\right) \\
& +\left(\left(f^{\prime}-g^{\prime}\right)^{*}\{u\}\right) \cdot g^{\prime *}\{v\}+g^{* *}\{v\} \cdot\left(\left(\left(f^{\prime}-g^{\prime}\right)^{*}\{u\}\right),\right.
\end{aligned}
$$

where we have denoted by $u$ and $v$ the cocycles of $P(n)$. Using the above formula inductively, we obtain

$$
\left(f^{\prime}-g^{\prime}\right)^{*}\left\{\mathbf{s}^{2}\right\}^{n+1}=\sum_{k=}^{n} 2^{k}\left(\begin{array}{l}
n \\
k
\end{array}\right)\left\{\lambda^{2}\right\}^{n+1-k}:\left\{g^{*} \boldsymbol{s}^{2}\right\}^{k},
$$

where $\lambda^{2}=\left(f^{\prime}-g^{\prime}\right)^{*} s^{2}=(f-g)^{*} s^{2}$. From (3.1) and (3.2), we get the conclusion of the Theorem.

Q. E. D.

Now, let two standard mappings $f, g: K^{2 n+1} \rightarrow P(n)$ be given such that $f\left|K^{2}=g\right| K^{2}$. Then we can construct a standard mapping $F$ as follows :

$$
\begin{aligned}
& F: \bar{K}_{0}^{2 n+1}+K_{1}^{2 n+1}+\bar{K}^{2} \times I \rightarrow P(n), \\
& F(x, 0)=f(x), F(x, 1)=g(x) \text { for } x \in \bar{K}^{2 n+1}, \\
& F(x, t)=f(x) \text { for }(x, t) \in K^{2} \times I ;
\end{aligned}
$$

where we have put $K_{i}^{: n+1}=K^{2 n+1} \times i(i=0,1)$. Then, from Lemma $1, F$ of (3.3) can be extended as follows :

$$
F^{\prime}: \bar{K}_{0}^{2 n+1}+\bar{K}_{1}^{2 n+1}+\bar{K}^{2 n} \times I \rightarrow P(n) .
$$

If we construct the obstruction $c^{2 n+2}\left(F^{\prime}\right)$ for such an extended $F^{\prime}$, this is evidently a cocycle in $K \times I-\left(K_{0}+K_{1}+L \times I\right)$. Then, from the construction, we get $F^{\prime *} s^{2}=f^{*} s^{2} \times(0,1)^{3)}$; and Theorem 1 shows that $\left\{c^{2 n+2}(F)\right\}$ is determined uniquely as a cohomology class in $K \times I-\left(K_{0}+K_{1}+L \times I\right)$, independently on the extension $F^{\prime}$. If we define

$$
c^{2 n+2}\left(F^{\prime}\right)=d^{2 n+1}(f, g) \times I,
$$

then the cohomology class $\left\{d^{2 n+1}(f, g)\right\}$ in $K-L$ is uniquely determined, and the correspondence $c^{2 n+2}(F) \rightarrow d^{2 n+1}(f, g)$ gives the isomorphism between the relative cohomology groups (see for ex. [3; Th. 25. 1]). We shall call $d^{2 n+1}(f, g)$ the separation obstruction. The following lemma is an easy consequence from the S.Eilenberg's theorem [1] and from the definition:

Lemma 2. If $f, g$ and $h: \bar{K}^{2 n+1} \rightarrow P(n)\left(\overline{K^{2 n+1}}=K\right)$ are standard mappings

3) If we regard $K \times I$ as a cellular complex composed of cells of the forms $\sigma^{k} \times 0, \sigma^{k} \times 1$ and $\sigma^{k-1} \times I$, then the $k$-cochains in $K \times I$ are only those of the forms $u^{k} \times \overrightarrow{0}, u^{k} \times 1$ and $u^{k-1} \times \bar{I}$, where $u^{k} \times \bar{O}\left(\sigma^{k} \times 0\right)=u^{k}\left(\sigma^{k}\right),=0$ otherwise etc. Then, these cochains have the following coboundaries:

$$
\begin{gathered}
\delta\left(u^{k} \times \overline{0}\right)=\delta u^{k} \times \overline{0}+(-1)^{k+1} u^{k} \times \bar{I}, \delta\left(u^{k} \times \overline{1}\right)=\delta u^{k} \times \bar{I}+(-1)^{k} u^{k} \times I, \\
\delta\left(u^{k} \times \bar{I}\right)=\delta u^{k} \times \bar{I} .
\end{gathered}
$$

Also, we have denoted $u^{k} \times \overline{0}+u^{k} \times \overline{1}=u^{k} \times(\overline{0}, \overline{1})$. 
such that $f\left|K^{2}=g\right| K^{2}=h \mid K^{2}$, then th? following propositions hold good :

$$
\begin{aligned}
& \left\{d^{2 n+1}(f, g)\right\}+\left\{d^{2 n+1}(g, h)\right\}=\left\{d^{2 n+1}(f, h)\right\} \\
& \left\{d^{2 n+1}(f, g)\right\}=0 \text { implies } f \simeq g \text { rel. } L^{4}{ }^{4}
\end{aligned}
$$

4. Classification theorem. Now, let $f, g: K^{2 n+1} \rightarrow P(n)\left(K=\bar{K}^{2 n+1}\right)$ be standard mappings such that $f\left|K^{2}=g\right| K^{\prime}$. When $f \simeq g$ rel. $L$, we can easily construct the standard homotopy connecting $f$ and $g$ as follows :

$$
\begin{aligned}
& F: K^{2 n+1} \times I \rightarrow P(n), \\
& F(x, 0)=f(x), \quad F(x, 1)=g(x) \text { for } x \in K^{2 n+1}, \\
& F(x, t)=f(x) \text { for } x \in L .
\end{aligned}
$$

In addition, we get from the assumption the standard mapping of (3.4):

$$
\begin{aligned}
& F^{\prime}: K_{0}^{2 n+1}+K_{1}^{2 n+1}+\bar{K}^{2 n} \times I \rightarrow P(n), \\
& F^{\prime}(x, 0)=f(x), F^{\prime}(x, 1)=g(x) \text { for } x \in K^{2 n+1}, \\
& F^{\prime}(x, t)=f(x) \text { for } x \in \bar{K}^{2} .
\end{aligned}
$$

Then, we obtain the following equations :

$$
\begin{aligned}
& F^{*} s^{2}=f^{*} s^{2} \times(0, \overline{1})-w^{1} \times I, \\
& F^{\prime *} s^{2}=f^{*} s^{2} \times(0, \overline{1}),
\end{aligned}
$$

where we have denoted by $w^{1}$ the homotopy cocycle constructed by the standard homotopy $F$, namely such a cocycle that takes an integer of $\pi_{2}(P(n)) \approx J$ represented by the mapping $F:\left(\left(\sigma^{1} \times I\right),\left(\sigma^{1} \times I\right)^{\bullet}\right) \rightarrow\left(S_{*}^{2}, e^{n}\right), \sigma^{1}$ being any $1-$ cell of $K$.

Now, when we apply Theorem 1 for $F^{\prime}$ and $F$, the left-hand side becomes to $\left\{d^{2 n+1}(f, g)\right\} \times I$ and the right-hand side becomes as follows :

This obviously reduces to

$$
\sum_{x=0}^{n} 2^{v}\left(\begin{array}{l}
n \\
k
\end{array}\right)\left\{w^{1} \times \bar{\Gamma}\right\}^{n+1-k} \cdot\left\{F^{*} s^{2}\right\}^{c}
$$

from the identities :

$$
2^{n}\left\{w^{1}\right\} \cdot\left\{f^{*} s^{2}\right\}^{n} \times I
$$

$$
0 \cdot 0=0,0 \cdot 1=0,1 \cdot 1=1, I \cdot 0=0, I \cdot 1=I, I \cdot I=0,
$$

and from the formulae of cup products in product complexes (see for instance [6]). Consequently, we obtain

$$
\left\{d^{2 n+1}(f, g)\right\}=2^{n}\left\{w^{1}\right\} \cdot\left\{f^{*} s^{2}\right\}^{n} .
$$

We shall prove that the condition (4.2) is also sufficient for the homotopy of $f$ and $g$. For this purpose, if there exists a cocycle $w^{1}$ in $K-L$ satisfying the condition (4.2) for two mappings $f, g: K^{2 n+1} \rightarrow P(n)$ such that $f\left|K^{2}=g\right| K^{2}$, we shall construct a standard mapping

$$
F: K_{0}^{2 i+1}+K_{1}^{2}+K^{1} \times I \rightarrow P(n)
$$

4) $f \cong g$ means that $f$ and $q$ are hom topic ; $f \simeq g$ rel. $L$ means that $f$ and $g$ are homotopic, fixing the image of $L$ unchanged during the homotopy. 
such that the conditions $F(x, 0)=f(x)$ and $(4.1)_{1}$ are fulfilled. Then from Lemma $1, F$ can be extended from $\bar{K}_{0}^{2 n+1}+\bar{K}^{2 n} \times I$ in $P(n)$; therefore, extending $F$ from $\bar{K}^{2 n+1} \times I$ in $P(n)$ by virtue of the homotopy extension theorem and denoting as $F(x, 1)=g^{\prime}(x)$, we get from the proposion (4.2) already proved, the following relation:

$$
\left\{d^{2 n+1}\left(f, g^{\prime}\right)\right\}=2^{n}\left\{w^{1}\right\} \cdot\left\{f^{*} s^{2}\right\}^{n} .
$$

Therefore we obtain from (3.5), $\left\{d^{2 n+1}\left(g, g^{\prime}\right)\right\}=0$, and also from (3.6) $g \simeq g^{\prime}$ rel. $L$, consequently $f \simeq g$ rel. $L$ holds. From these facts we obtain

THEOREM 2. Let $f, g: K^{2 n+1} \rightarrow P(n),\left(K^{2 n+1}=K\right)$ be standard mappings such that $f\left|\bar{K}^{2}=g\right| K^{2}$. Then $f \simeq g$ rel. $L$ if and only if there exists an integral cocycle $w^{1}$ in $K-L$ such that

$$
\left\{d^{2 n+1}(f, g)\right\}=2^{n}\left\{w^{1}\right\} \cdot\left\{f_{-}^{*} s^{2}\right\}^{n} .
$$

This is an extension of the L. Pontrjagin's result.

5. Covering maps. We have remarked that $S^{2 n+1}$ is a circle bundle having the base space $P(n)$. Therefore, when a continuous mapping $f$ from the complex $K$ in $P(n)$ is given, we can construct from this an induced orientable circle bundle $B$ having the base space $K[4 ; \mathrm{p} .47]$. Namely, if we denote by $\phi_{i}$ the coordinate function of $P(n)\left(\left\{U_{i}\right\}\right.$ being the covering of $P(n)$ which constitute coordinate neighborhoods for $\left.S^{2 n+1}\right)$, we can define a coordinate function $\psi_{i}$ for $B$ with the coordinate neighborhoods $\left\{V_{i}\right\}$ $\left(V_{i}=f^{-1}\left(U_{i}\right)\right)$ as follows :

$$
\psi_{i}(x, t)=\psi_{i}, x_{x}(t)=x \times \phi_{i}(f(x), t) \text { for } x \in V_{i}, t \in S^{1} .
$$

(See for ex. [4; p. 48]). Then we can construct a continous mapping $h: B \rightarrow S^{2 n+1}$ by the formula

$$
h(b)=\phi_{i}\left(f p(b), p_{i}(b)\right), \quad b \in B, p(b) \in V_{i},
$$

denoting by $p$ the projection of $B$, and by $p_{i}: q^{-1}\left(V_{i}\right) \rightarrow S^{1}$ the mapping $\psi_{i, x}^{-1}$. In such circumstances the commutativity of the mappings holds good:

$$
p_{n} h=f p .
$$

Now, we shall calculate the characteristic cohomology class $[4 ;$ p. 178$]$ for the bundle $B$. For this purpose, we can assume without any loss of generality, that $f$ is a standard mapping [4: p.53]. On the other hand, the desired class is nothing but the image by $f^{\prime \prime}$ of the characteristic cohomology class of $S^{2 n+1} \quad$ [4; p. 179]; and from the standardness of $f$ we may only calculate in $S_{*}^{3}$. This is easily proved to be $\pm s^{2}[5]$. Therefore the required cohomology class must be $\pm\left\{f^{\prime \prime} s^{2}\right\}$. (Cf. also [4;p. 153]).

Now, we shall call the continuous mapping $f: K \rightarrow P(n)$ to be algebraically inessential provided there exists a standard mapping $f^{\prime}: K \rightarrow P(n)$ homotopic to $f$ and such that $\left\{f^{\prime *} s^{2}\right\}=0$. Then, from the above arguments, we can see that the characteristic cohomology class of the bundle $B$ induced by an algebraically inessential mapping must be zero. From this fact and from 
the trivialities of $\pi_{k}\left(S^{1}\right)(k \geqq 2)$, we can easily conclude that $B$ admits a cross-section $[4 ;$ p. 178]:

$$
\xi: K \rightarrow B, p \xi=\text { identity. }
$$

Therefore (5. 2) asserts the existence of the mapping $\eta_{f}: K \rightarrow S^{2 n+1}$ such that :

$$
\eta_{f}=h \xi ; p_{n} \eta_{f}=p_{n} h \xi=f p \xi=f .
$$

Consequently, we get the following theorem :

THEOREM 3. Let $f: K \rightarrow P(n)$ be an algebraically inessential mapping. Then there exists a mapping $\eta_{f}: K \rightarrow S^{2 n+1}$ such that $p_{n} \eta_{f}=f$.

6. Another classification theorem. The purpose of the preceding and this sections is to prove the following classification theorem:

THEOREM 4. Let the 1-dimensional integral cohomology group of $K$ be zeso. Then the homotopy classes of all the algebraically inessentital mappings from the complex $K$ into $P(n)$ correspond 1-1 way with those of the mappings from $K$ in $S^{2 n+1}$; and the correspondence is induced by $\dot{p}_{n}$. When $n=1$, the cohomological assumption can be removed.

PROOF. It is sufficient to prove that for two algebraically inessential mappings $f, g: K \rightarrow P(n)$ such that $f \simeq g, \eta_{f} \simeq \eta_{g}$ holds good for the mappings $\eta_{f}, \eta_{g}: K \rightarrow S^{2 n+1}$ constructed by Theorem 3 . Now, the covering homotopy theorem shows the existence of the mapping $\eta: K \rightarrow S^{2 n+1}$ such that

$$
\eta_{f} \simeq \eta, \quad p_{n} \eta=g=p_{n} \eta_{g} .
$$

Therefore, it is sufficient to prove only $\eta \simeq \eta_{g}$. Now, let $\left\{V_{i}\right\}$ be the coordinate neighborhoods such that $V_{i}=g^{-1}\left(U_{i}\right)$ for the coordinate neighborhoods $\left\{U_{i}\right\}$ for $S^{2 n+1}$. Then, $\eta$ and $\eta_{g}$ can be expressed as follows:

$$
\begin{aligned}
& \eta(x)=\phi_{i}\left(g(x), p_{i} \eta(x)\right) \\
& \eta_{g}(x)=\phi_{i}\left(g(x), p_{i} \eta_{g}(x)\right)
\end{aligned} \quad \text { for } x \in V_{i} .
$$

Now, let $B$ be an induced bundle by $g$, and let $\psi_{i}$ be its coordinate function constructed by the same method quoted in the preceding paragraph.

Then, we can construct the mappings $\eta, \eta_{g}: K \rightarrow B$ by the formulae:

$$
\begin{aligned}
& \eta(x)=\psi_{i}\left(x, p_{i} \eta(x)\right) \\
& \eta_{g}(x)=\psi_{i}\left(x, p_{i} \eta_{g}(x)\right)
\end{aligned} \quad \text { for } x \in V_{i}
$$

Here, we can easily see that $\eta$ and $\eta_{g}$ are cross-sections. We can also prove that these cross-sections are homotopic, from the assumption and from the theorem of N. E. Steenrod [4; Th. 34. 8, p. 176] as $\pi_{k}\left(S^{1}\right)$ vanish for $k \geqq 2$. When we denote by $\zeta: K \times I \rightarrow B$ the homotopy connecting $\eta$ and $\overline{\eta_{g}}$, then $h \zeta: K \times I \rightarrow S^{2 n+1}$ is a homotopy connecting $\eta$ and $\eta_{g}$, denoting by $\boldsymbol{h}: B \rightarrow S^{2 n+1}$ the bundle mapping defined analogously to (5.1).

If $n=1$, the assumption $H^{1}(K, J)=0$ can be removed on account of 
the fact that $S_{*}^{3}$ becomes a group space [2].

Q. E. D.

REMARK. The results of $\S \S 5,6$ can be extended to the relative case, where the subcomplex $L$ is mapped to a single point of $P(n)$; but it is too artificial, and would be unnecessary to be stated.

\section{BIBLIOGRAPHY}

[1] S. Eilenberg, Cohomology and continuous mappings, Ann. of Math., 41 (1940), 231-251.

[2] L. Pontrjagin, A classification of mappings of the three-dimennsional complex into the two dimensional sphere, Rec. Math. (Mat. Sbornik) N.S. $9(51)(1941), 331-363$.

[3] N. E.STEENROD, Products of cocycles and extensions of mappings, Ann. of Math., 48 (1947), 290-320.

[4] N.E.Steenrod, The topology of fibre bundles, Princeton 1951.

[5] H. WADA, On the structure of a sphere boundle, Tôhoku Math. I. 2nd ser. 3 (1951), 136-139.

[6] H. Whitney, On products in a complex, Ann. of Math., 39 (1938), 397-432.

Mathematical Institute, TôHOKu UnIVERsity, Sendai. 